\title{
Boundary Exposure Using Intensity and Texture Gradient Features
}

\author{
Ms.S.Veeralakshmi ${ }^{1}$, Mrs.S.Vanitha Sivagami ${ }^{2}$, Ms.V.Vimala Devi ${ }^{3}$, \\ Ms.R.Udhaya ${ }^{4}$ \\ ${ }^{1,4}$ (Assistant Professor, Department Of Information Technology), \\ 1,3,4 (P.S.R.Rengasamy College of Engineering for Women, Sivakasi, Tamil Nadu, India) \\ ${ }^{2}$ (Assistant Professor in Computer Science and Engineering) \\ ${ }^{3}$ (Assistant Professor, Department Of Computer Science and Engineering) \\ ${ }^{2}$ (Mepco Schlenk Engineering College, Sivakasi, Tamilnadu)
}

\begin{abstract}
Images are used in many fields, including surveillance, medical diagnostics and non-destructive testing. Edge detection and boundary detection plays a fundamental role in image analysis and computer vision. A boundary map of image can provide valuable information for further image analysis and interpretation tasks such as segmentation, object description etc. Boundaries are mainly used to detect the outline or shape of an object. Image segmentation is used to locate objects and boundaries in images and it assigns a label in every pixel in an image such that pixels with the same level share have certain virtual characteristics. The paper proposed an edge detection technique for detecting the correct boundary of objects in an image. It can detect the boundaries of object using the information from intensity gradient using the vector image model and texture gradient using the edge map model. The proposed method for edge following technique is efficient and it is applicable on various kinds of medical images such as ultrasound images, magnetic resonance (MR) images, and computerized tomography images.
\end{abstract}

Index Terms: Vector image model, Edge mapping model, Edge detection technique, Boundary detection

\section{Introduction}

Boundary detection is mainly used to detect the outline or shape of the object, so we can easily identify objects based upon the outline or shape. Segmentation is the process in which an image is divided into its constituent objects or parts. The main goal of segmentation is to simplify and/or change an image representation into something that is analyzed easily. Image segmentation is mainly used to locate objects and boundaries (lines, curves, etc) in images and it is the process of assigning a label to every pixel in an image. The result of image segmentation is a set of segments that collectively covers the entire image, or a set of contour extracted from the image.

The main goal of image segmentation is to cluster the pixels into salient image regions, i.e., regions are corresponding to objects, or natural parts of objects. Image segmentation can be secret into two types.

1. $\quad$ Edge based approach

2. Region based approach

\subsection{Edge based approach}

Edge based methods is to use an edge detection operator such as Robert, Sobel, Prewitt, Laplacian and Canny which is used for detecting the object boundaries and then extract the boundaries by using edge information. Edge based methods center around contour detection.

The weakness of edge based method is resulting regions may not be connected; hence edges need to be joined and prone to failure in the presence of blurring.

\subsection{Region based approach}

Region based methods are partitioning an image into connected regions and by grouping neighboring based on image properties. The image properties consist of, the intensity values from the original images or values are computed based on an image operator and texture are unique to each region. Region based methods are based on similarity of regional image data. Some of the most widely used approaches are clustering, region growing, and region splitting and region merging. 


\section{Related Work}

The existing method for some boundary detection techniques are Active Contour Model (ACM), Active Contour without Edges (ACWE), Geodesic Active Contour (GAC), Gradient Vector Flow (GVF), Vector Field Convolution (VFC).

M. Kass et al. [2] proposed an Active Contour Model. A framework of various methods for image and video segmentation is known as active contour. Active Contour Models have been one of the most successful methods for image segmentation. Active Contour Model deforms a contour to lock onto features of curiosity within an image. Usually the features are lines, edges and/or object borders. Active contour models should be able to find the boundary in given images. Snakes or Active Contours are used extensively in image processing application and computer vision; particularly it is used to locate object boundaries. For example, a snake is used to automatically find a manufactures part of an assembly line; one might be used to find the outline or shape of an image. The main disadvantage of active contour model is the resulting of image is poor quality of image.

Chan-Vese et al. [3] proposed an Active Contour without Edges (ACWE), it is the minimization of energy based segmentation and it is used to detect the shape of objects in a given image based on curve evolution: starting with an initial curve and evolving it to the 'correct' steady state, i.e. the object boundaries. The disadvantage of ACWE is detecting the boundaries that are not necessarily defined by gradient.

V. Caselle and R. Kimmel et al [4] proposed a Geodesic Active Contours (GAC). It is used to detect the boundaries of object and deal with geometric information of image. The geodesic active contour is based on theory of curve evolution and it is represented as the zero level-set of a 3D function, the geodesic curve computation is used to reduce a geometric flow that is similar to curve evolution. The limitation of GAC method is that a contrast constant should be selected in the edge stop function.

C. Xu and J. L. Prince et al [5] proposed a Snakes, Shapes, and Gradient Vector Flow. It describes snake and active contours, which are used to locate the object boundaries. An external force for snake is called gradient vector flow (GVF), is computed a diffusion of the gradient vectors of a gray-level or binary edge map derived from the image and the resultant field has a large capture range and forces active contours into concave regions. An active contour is used in GVF field as its external force is called GVF snake. The main advantages of the GVF snake over a traditional snake are its insensitivity to initialization and ability to move into concave boundary regions. The main limitation of GVF method is that the parameters are very sensitive.

B. Li et al [6] proposed a Vector Field Convolution model; it is used to calculate the external force for deformable models, including active contours and deformable surfaces. An external force for active models called Vector Field Convolution (VFC). This external force is calculated by convolving a vector field with the edge map derived from image. Active contours that use vector field convolution external force are termed VFC snakes and the VFC snakes are constructed by a way of a force balance condition. The main drawback of vector field convolution is that the weak edges might be overwhelmed by the strong edges along with the noise.

In proposed technique can detect the boundaries of objects in the images. It detects the boundaries of object using the information from the intensity gradient using the vector image model and the texture gradient using the edge mapping model. The proposed edge detection technique is based on the vector image model and the edge mapping model. The edge vector gradient model provides a more complete description of an image. It provides a complete description of an image based on both directions and magnitudes of image edges. The main objective of proposed method is to perform the boundary detection of images for such as medical, noise and disease affected on a particular magnetic resonance (MR) and computerized tomography (CT) images.

\section{Proposed System}

In proposed boundary detection algorithm is used to detect the boundary of object in an image. Boundary extraction algorithm consists of following three phases.

1. edge vector gradient

2. Edge mapping model

3. Edge detection Technique

\subsection{Edge Vector Gradient Calculation}

Proposed technique relies on detecting the direction and magnitude for a given input image and it is found to be sufficient for boundary detection. To determine the boundary in an image is use the boundary extraction algorithm.

Edge vector gradient is used to perform vector operations on an image. Vector defines the direction and magnitude of the image. For an image the edge vector field is calculated from

$$
\vec{v}(i, j)=\frac{1}{n}\left(C_{x}(i, j) \vec{i}+C_{y}(i, j) \vec{j}\right)
$$




$$
\begin{array}{r}
\vec{v} \approx \frac{1}{n}\left(\frac{\partial f(x, y)}{\partial y} \vec{i}-\frac{\partial f(x, y)}{\partial x} \vec{j}\right) \\
n=\max _{i, j}\left(\sqrt{C_{x}(i, j)^{2}+C_{y}(i, j)^{2}}\right)
\end{array}
$$

Here, $\mathrm{C}$ is the convolution between the input image and corresponding mask difference, $\mathrm{n}$ is the maximum number of pixels moving towards either vertical direction or horizontal direction.

$$
\begin{aligned}
& C_{x}(i, j)=-G_{y} \times f(x, y) \\
& C_{y}(i, j)=G_{x} \times f(x, y)
\end{aligned}
$$

Where $G_{x}$ and $G_{y}$ are gaussian difference mask. Edge vector of an image is to indicate the magnitude and direction of edges and form a vector stream flowing around an object. The magnitude and direction are calculated from, i.e.,

$$
\begin{array}{r}
M(i, j)=\frac{1}{C_{r}} \sum_{(i, j) \varepsilon N} \sqrt{C_{x}(i, j)^{2}+C_{y}(i, j)^{2}} \\
D(i, j)=\frac{1}{C_{r}} \sum_{(i, j) \varepsilon N} \tan ^{-1}\left(\frac{C_{y}(i, j)}{C_{x}(i, j)}\right)
\end{array}
$$

Where $\mathrm{C}_{\mathrm{r}}$ is the total number of pixels in the neighborhood $\mathrm{N}$. The $3 \times 3$ window as the neighborhood $\mathrm{N}$.

\subsection{Edge Mapping Model}

The edges of object in an image can be detected by using edge mapping model. The vector image model and the edge mapping model are applied to select the best edges. The Proposed method yields more descriptive vectors along the object edge than that of the original edge vector field. This is useful for boundary extraction of objects in images.

Edge mapping model is derived from law's texture and canny edge detection. It gives important information of the boundary of object in images.

\subsubsection{Law's texture}

Law's texture is a one type of texture filter; it is applied to the image to create the filtered image from which texture features are computed. Law's texture is computed by convolving an input image with each of the masks.

\section{Law's texture masks}

$$
\begin{array}{ll}
\text { L5 (Level) } & = \\
\text { E5 (Edge) } & =\left[\begin{array}{lllll}
1 & 4 & 6 & 4 & 1
\end{array}\right] \\
\text { S5 (Spot) } & =\left[\begin{array}{lllll}
-1 & -2 & 0 & 2 & 1
\end{array}\right] \\
\text { R5 (Ripple) } & =
\end{array}
$$

Where,

(L5) (Gaussian) gives a center-weighted local average

(E5) (Gradient) responds to row or column step edges

(S5) (LOG) detects spots

(R5) (Gabor) detects ripples

\section{Creation of 2D masks}

1D mask are multiplied to construct 2D masks: For example mask E5L5 is the product of E5 and L5. 


$$
\text { E5 }\left(\begin{array}{c}
-1 \\
-2 \\
0 \\
1
\end{array}\right) \times\left(\begin{array}{lllll}
1 & 4 & 6 & 4 & 1
\end{array}\right)=\left(\begin{array}{cccccc}
-1 & -4 & -6 & -4 & -1 \\
-2 & -8 & -12 & -8 & - \\
0 & & & \\
0 & 0 & 0 & 0 & 0 \\
2 & 8 & 12 & 8 & 2 \\
1 & 4 & 6 & 4
\end{array}\right)
$$

\subsubsection{Canny edge detection}

\section{E5L5}

Canny edge detection is used to detect the edges in an image. It involves the following four steps.

1. Convolution with Gaussian filter

2. Calculate the magnitude and direction of gradient

3. Non-maximal suppression

4. Thresholding

The first step of canny edge detection is convolution; it is used to reduce the noise in given input image. The second step calculates the magnitude or direction of the gradient. The third step is non-maximal suppression to identify the edges. The fourth step is thresholding to detect and link the edges.

\subsection{Edge Detection Technique}

The boundary of an object can be detected by using edge following technique. It detects the boundary of object using the information from edge vector gradient and edge mapping model and it is used for searching more information about correct boundary of an object. At the position (i, j) of an image, the position of edges can be calculated by $3 \times 3$ matrix

$$
\begin{aligned}
L_{i, j}(r, c)=x M_{i, j}(r, c)+y D_{i, j}(r, c)+z E_{i, j}(r, c) & \\
& 0 \leq \mathrm{r} \leq 2, \quad 0 \leq \mathrm{c} \leq 2
\end{aligned}
$$

Where $\mathrm{x}, \mathrm{y}$, and $\mathrm{z}$ are weight parameters that control the edge to flow around an object. An element in the $L_{i . j}$ indicates the strong edge in the corresponding direction. The $3 \times 3$ matrices $M_{i, j}, D_{i, j}, E_{i, j}$ is calculated as follows:

$$
\begin{gathered}
M_{i, j}(r, c)=\frac{M(i+r-1, j+c-1)}{\max _{i, j} M(i, j)} \\
D_{i, j}(r, c)=1-\frac{|D(i, j)-D(i+r-1, j+c-1)|}{\pi} \\
E_{i, j}(r, c)=E(i+r-1, j+c-1)
\end{gathered}
$$

Where $M(i, j)$ is indicate the average magnitude of edge vector gradient field, $D(i, j)$ is the direction of edge vector field and $E(i, j)$ is the edge map.

The values of each element in the matrices are ranged between 0 and 1 . Edge detection technique to identify the flow direction at each pixel location to the closest boundary; then followed by the direction of locations that encounter two opposite directions of edge flow. If any of the image attribute such as color, texture, or other combination can be used to compute the edge energy and direction of flow, this scheme provides a general framework for boundary detection.

\section{Results And Discussions}

The proposed method has been implemented and tested on various digital images. The following results are obtained.

Figure 5.1 (a) shows the input head in MR image. Figure 5.1 (b) shows the calculation of edge vector gradient model; it gives the direction and magnitude of the images. The vector is defined as arrows and the 
arrows indicate the direction of moving pixels towards either vertical direction or horizontal direction. Figure 5.1 (c) shows the law's texture, it convolutes the input image and 2D mask. The output of convoluted images is given as input to canny edge detection. Figure 5.1 (d) shows the edge detection using canny edge detection; it detects the edges for the given input image. Figure 5.3(e) shows the boundary detection using edge detection technique. Edge detection technique is used to detect the boundary using the information from intensity gradient using vector image model and texture gradient using edge mapping model. The vector image model provides complete description of images considering both direction and magnitude of image edges. Map the edge model is used to detect the edges in an image. The vector image model and edge mapping model is used to select the best edges in an image.

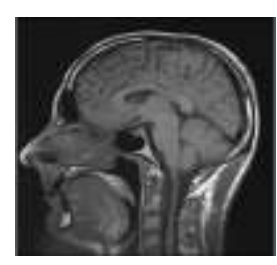

(a)

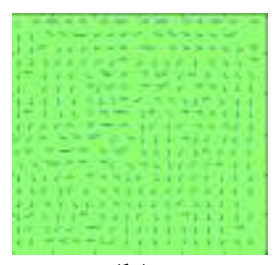

(b)

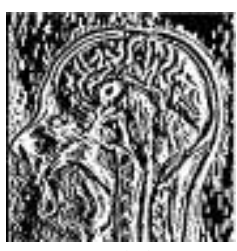

(c)

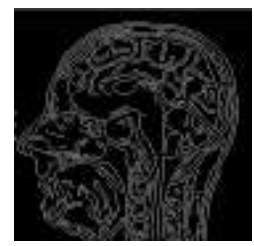

(d)

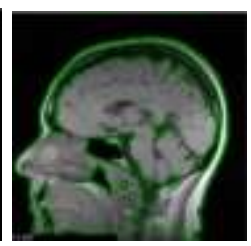

(e)

Figure5.1 (a) Head in MR image (b) Calculate Edge vector gradient (c) Law's texture (d) Detected edges using canny edge detection (e) Boundary detection using Edge detection technique

These implementations are applied to disease affected medical images. Figure5.2 shows the boundary detection for disease affected image. For example if brain is affected by cancer cells, the proposed method detects the particular cancer cells spreading area in brain image.

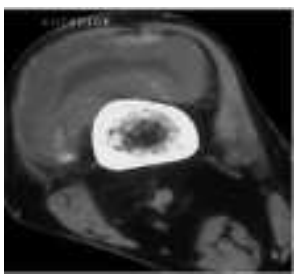

(a)

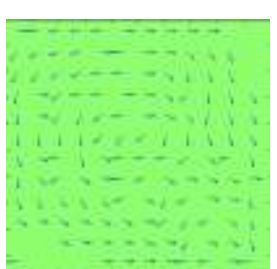

(b)

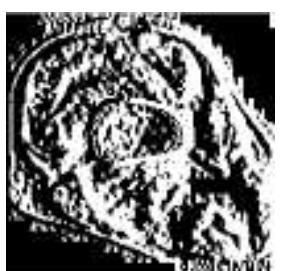

(c)

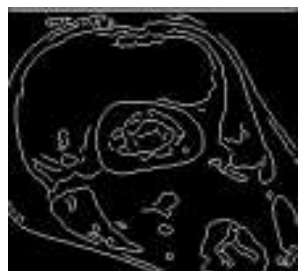

(d)

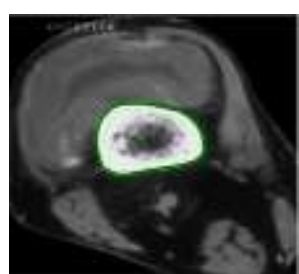

(e)

Figure5.2 (a) Disease affected image (b) Calculate edge vector gradient (c) Law's texture (d) detected edges using canny edge detection (e) Boundary detection for disease affect of an image

\subsection{Comparision Analysis}

Figure 5.3 shows the comparison between the obtainable Geometric Active Contour (GAC) model and proposed method (Edge following technique). GAC model only detects the outline of the object, but the proposed method is to detect the boundary of object including the inner parts.

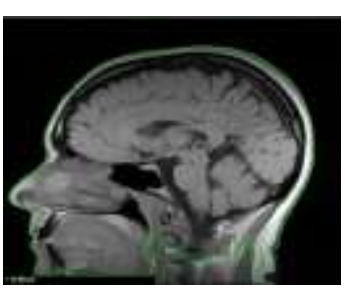

(a)

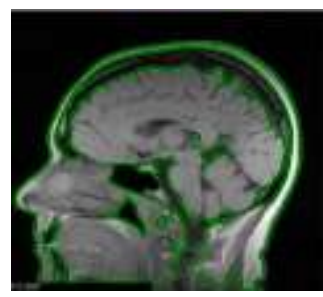

(b)

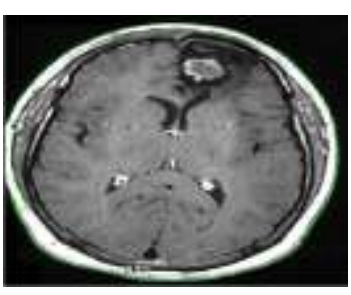

(c)

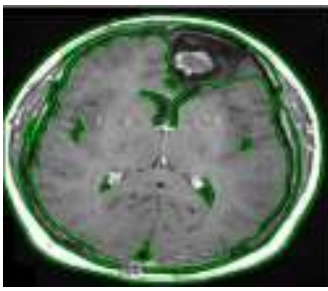

(d)

Figure 5.3 (a) \& (b) Geometric active contour (c) \& (d) Edge detection technique (Proposed method)

\section{2 performance evaluation}

Table1 shows average CPU computation time (in seconds) for GAC model and proposed model. These two models are executed in same machine configuration. Comparing to GAC model, proposed method take less computation time for detecting the boundary of the object in an image. The main reason for reduced computation time in proposed method is using the Sussman method. Sussman method uses CFL (Courant Friedrichs lewy) condition; it collects boundary detection related information from vector image model and map the edge model. The GAC model use Neumann boundary condition method, it fails to accurately identify the pixel location, so the proposed method is more efficient comparing to the GAC model. 
Table1: Average CPU computation time (in seconds) for Geometric Active Contour (GAC) model and proposed method (Edge detection technique)

\begin{tabular}{|c|c|c|}
\hline Methods & $\begin{array}{c}\text { GAC (Geometric Active } \\
\text { Contour) }\end{array}$ & Proposed Model \\
\hline Medical image database & 102.01 & 35.51 \\
\hline $\begin{array}{c}\text { Boundary detection for } \\
\text { disease affected } \\
\text { Images }\end{array}$ & 18.14 & 5.27 \\
\hline
\end{tabular}

[1]. Web Reference (www.imaging.consult.com)

\section{Conclusion}

Normally images are used in many fields including surveillance, medical field and non destructive testing. Edge map and boundary detection are important for image analysis and some interpretation task such as object segmentation, object registration and object description etc. The proposed method in edge detection technique is used to detect the correct boundary of object in an image. It detects the correct boundary of object using the information from vector image model and edge mapping model. The edge vector gradient model provides the complete description about the image and these two models are used to select the best edges in an image. The proposed method is more efficient to detect the correct boundary of object comparing to the existing model. Comparing to the existing model, our proposed method is take less computation time for detecting boundary of objects. The proposed method is not only applicable for medical image; it can be applied to any image processing problems in which ill defined boundaries are detected.

\section{Acknowledgement}

We sincerely thank our college, Mepco Schlenk Engineering College for humble facilities and necessary infrastructure made available during the course of our work. We wish to express our thanks and sincere gratitude to our Principal and Head of the Department for his guidance to complete this work successfully and enthusiastic encouragement.

\section{References}

[1] Krit Somkantha, Nipon Theera-Umpon, and Sansanee "Boundary Detection in Medical Images Using Edge Following Algorithm Based on Intensity Gradient and Texture Gradient Features”, IEEE Transaction On Biomedical Engineering . Vol. 58, No. 3, March 2011.

[2] M. Kass, A. Witkin and D. Terzopoulos "Snakes: Active contour models", Int. J. Comput. Vis., vol. 1, pp.321 - $331,1987$.

[3] T. Chan and L. Vese "Active contours without edges", IEEE Trans. Imag. Process, vol. 10, no. 2, pp.266 - $277,2001$.

[4] V. Caselles, R. Kimmel and G. Sapiro "Geodesic active contours", Int. J. Comput. Vis., vol. 22, no. 1, pp.61 - 79, 1997.

[5] C. Xu and J. L. Prince "Snakes, shapes, and gradient vector flow", IEEE Trans. Imag. Process, vol. 7, no. 3, pp.359 - 369 , 1998.

[6] B. Li and T. Acton "Active contour external force using vector field convolution for image segmentation", IEEE Trans. Imag. Process, vol. 16, no. 8, pp.2096 - 2106, 2007.

[7] N. Eua-Anant and L. Udpa "A novel boundary extraction algorithm based on a vector image model", Proc. IEEE Sym. Circ. and Syst., pp.597 - 600, 1996.

[8] J.-D. Lee, H.-R. Su, P. E. Cheng, M. Liou, J. Aston, A. C. Tsai and C.Y. Chen "MR image segmentation using a power transformation approach", IEEE Trans. Med. Imag., vol. 28, no. 6, pp.894 - 905, 2009.

[9] J. Carballido-Gamio, S. J. Belongie and S. Majumdar "Normalized cuts in 3-D for spinal MRI segmentation", IEEE Trans. Med. Imag., vol. 23, no. 1, pp.36-44, 2004. .

[10] J. F. Canny "A computational approach to edge detection", IEEE Trans. Pattern Anal. Mach. Intell., vol. PAMI-8, no. 6, pp.679 698, 1986.

[11] Chunming Li, Chiu-Yen Kao, John C. Gore, and Zhaohua Din "Minimization of Region - Scalable Fitting Energy for Image Segmentation", IEEE Transaction on Image Processing, vol. 17, no. 10, October 2008.

[12] V. Caselles, F. Catte, T. Coll and F. Dibos "A geometric model for active contours", Numer. Math., vol. 66, pp.1 - $31,1993$.

[13] F. Leymarie and M. D. Levine "Tracking deformable objects in the plane using an active contour model", IEEE Trans. Pattern Anal. Mach. intell. vol. 15, no. 6, pp.617 - 634, 1993.

[14] C. Xu and J. L. Prince "Gradient vector flow: A new external force for snake", IEEE Proc. Conf. Comput. Vis. Pattern Recognition., pp. 66 - 71, 1997.

[15] R. C. Gonzalez and R. E. Woods Digital Image Processing. Reading. 1992. : Addison-Wesley

[16] G. S. Robinson "Edge detection by compass gradient masks", Comput. Graph. Image Process. vol. 6, no. 5, pp.492 - 501,1977. E. Argyle "Techniques for edge detection", Proc. IEEE, vol. 59, no. 2, pp.285 - 287, 1971.

[17] J. Cheng and S. W. Foo "Dynamic directional gradient vector flow for snakes", IEEE Trans. Imag. Process. , vol. 15, no. 6, pp.1563 $-1571,2006$.

[18] A. Guocheng, C. Tianjin and W. Zhenyang "A fast external force model for snake-based image segmentation", Proc. Int. Conf. Signal Process., pp.1128 - 1131, 2008.

[19] [online] Available: http://mrcas.mpe.ntu.edu.sg/

[20] [online] Available: http://icsl.washington.edu/node/316

[21] [online] Available: http://imaging.consult.com 